\title{
The complexity of planning for goods delivery in a shared urban space: a case study involving cyclists and trucks
}

\author{
Kelly Pitera $^{1}{ }^{1} \cdot$ Petr Pokorny $^{1} \cdot$ Terje Kristensen $^{2} \cdot$ Astrid Bjørgen $^{2}$
}

Received: 3 January 2017 / Accepted: 11 August 2017 / Published online: 19 August 2017

(C) The Author(s) 2017. This article is an open access publication

\begin{abstract}
Introduction Growth in urban areas has resulted in conflicts between road users as they share the roadway. Such conflicts are only exacerbated by failings in the planning process. The purpose of this study is to examine, through a case study in Trondheim, Norway, issues related to freight delivery on a street section with a high volume of cyclists in order to identify how and where urban freight should be addressed within the urban planning process.

Methods The study consists of two parts: (1) a safety evaluation of the location through video recordings which identifies the risk areas associated with freight delivery and encounters with cyclists, and (2) a mapping of the planning process through semi-structured interviews in order to understand the development decisions involving a facility requiring deliveries and the surrounding streetscape.

Results The safety analysis identified several risk areas mainly associated with the turning manoeuvres of trucks undertaken either before or after deliveries. The mapping of the planning process indicated that there were few to no discussions of freight deliveries during the planning, design and construction of the building; neither were there any for the streetscape projects taking place within the same time frame.
\end{abstract}

This article is part of Topical Collection on Accommodating urban freight in city planning

Kelly Pitera

kelly.pitera@ntnu.no

1 Department of Civil and Environmental Engineering, Norwegian University of Science and Technology (NTNU),

7491 Trondhem, Norway

2 SINTEF Technology and Society, Postboks 4760 Sluppen, 7049 Trondheim, Norway
Conclusions The absence of a dedicated freight policy and/or personnel at the governing authority, as well as lack of coordination amongst different plans (construction, street, thematic), are likely to lead to continued problematic and potentially dangerous environments such as that in the case study. Improvements to the Norwegian planning process, namely earlier integration of freight considerations, are required to ensure sustainable freight systems in the urban environment.

Keywords Freight delivery $\cdot$ Urban freight $\cdot$ Safety $\cdot$ Bicycles

\section{Introduction}

As a result of growing urban areas, both competition for space and conflicts between road users are increasing. One area of notable concern are the encounters between freight vehicles and cyclists, both whose numbers are rising in many cities. Given the size and mass differential of these two road users, their interactions can result in severe consequences for cyclists [1-3]. Within the European Union, $12 \%$ of cyclist fatalities in 2013 were the result of an accident involving a truck [4], while within Norway this number is nearly $20 \%$ country-wide, and $35 \%$ when specifically considering urban areas [5]. Given such consequences, the co-existence of trucks and bicycles in complex urban areas has started to attract research attention focusing mainly on accident analysis and detection of cyclists in blind spots [5-7]. More complex analysis, like that related to urban planning and design, still needs to be investigated further.

Having goals such as creating attractive and liveable cities, urban development is focused on acknowledging and facilitating different users and activities on shared urban streets. The freight community has identified the potential for conflicts between trucks and non-motorized users as a concern in urban areas [8-11], but this concern has been addressed in 
limited ways within practice. Within many freight plans and guidelines of urban freight best practices, including those specific to the location studied in this research, there is little mention of accommodations for sharing the street with nonmotorized users such as bicycles, or more specifically, how to design infrastructure to do this. For example, the BESTUFS Good Practice Guide on Urban Freight Transport [12] discusses guidance on goods, vehicle access and loading, but does not mention safety or the need to share infrastructure with different users. Guidance from the Norwegian national level regarding goods deliveries in cities identifies issues associated with freight deliveries, even discussing concerns related to cyclists, among others, sharing street space with trucks. It is stated that for new establishments, it is important to look at the road network and consider the type of conflicts that may occur, specifically mentioning bicycle lanes as an area of concern; however, there is no additional guidance on how to mitigate for such potential conflict [13]. This is similar at the local level. For example, the street use plan for Trondheim [14] recommends that the city provide good and safe delivery conditions in new buildings, but there is no follow-up with specific suggestions as to what this may entail.

Because freight transport is one of the primary users of the urban space, several European cities are now working on developing Sustainable Urban Logistics Plans (SULP) [15]. In order to improve conditions for local freight delivery, the primary objective of an SULP is to enhance the cooperation and predictability between the different actors within urban logistics. Previous projects (e.g. the Enclose Project, see [16] have made attempts at creating guidelines for developing and implementing SULPs. Key components of the guidelines include: setting objectives and targets; identifying the logistics context, including identifying key actors and assessing their requirements for improving city logistics; identifying policy measures and service designs which can be analyzed and assessed; assignment and distribution of responsibilities; and development of implementation and monitoring plans. The interest in developing SULPs has also risen in Norway, and several Norwegian industry representatives have recently proclaimed an urgent need for implementing logistics plans in Norwegian cities [17]. SULPs have the potential to help decision makers and planners/designers to better understand and address the trade-offs and conflicts between users of shared urban spaces; however, more work is required to understand the complexity of urban freight delivery and how laws and regulations affect present systems.

To demonstrate the complexity and challenges with planning effective freight logistics in an urban environment, we conducted a case study in Trondheim, Norway. The selected case, which centres on the planning, construction, and operation of a typical grocery store, illustrates a typical case for developers, planners, and users in urban development. While Trondheim is a medium-size city with nearly 200,000 inhabitants, it has a large bicycle modal share of $9 \%$, which is the highest of all cities in Norway [18], and strongly promotes initiatives to construct and improve bicycle infrastructure in the city. Similar to many small to medium-size urban areas, the city of Trondheim does not have a specific plan for urban freight logistics, but has expressed an interest in developing such a plan.

This study considers the planning and design of a building site having a requirement for truck deliveries, which coincided with the planning and design of a cycling facility in the same location. Observations of the site post-construction have identified safety risks associated with truck deliveries intensified by the high bicycle volumes along the street. It is our hypothesis that the complex urban environment, consisting of overlapping yet often uncoordinated domains, as well as lack of a dedicated freight plan and/or planner, contributed to this situation. The study uses multiple methods to illustrate the lack of focus on freight-related issues within the urban planning process. In combining methods through a common case study, both the cause and the effect are considered together, further emphasing the consequences of uninformed decision-making within urban planning and design.

The article begins with a description of the case study. This is followed by a description and discussion of results of the safety analysis, which consisted of both video observations and an intercept interview. The safety analysis identifies risks associated with good delivery at the site, clearly illustrating the adverse impacts of a disconnect between urban logistics planning and urban mobility planning. The second part of the analysis follows, focusing on the planning process of the site. Through document analysis and semi-structured interviews, the planning process is mapped to identify deficiencies concerning inclusion of urban freight which may have contributed to the risky delivery conditions identified. The article concludes with a discussion integrating the results of both analyses and suggests solutions to mitigate these issues in the future.

\section{Case study description}

This case study focuses on a section of roadway in front of a grocery store located in a moderately dense mixed-use area in Trondheim, Norway. The site is representative of grocery stores in mixed-use areas within Trondheim, both with regard to the delivery demands of the store and to the movements and activity on the streets in the vicinity of the store. The grocery store shares a building with an academic institution and is located along an important part of Trondheim's cycling network. Additionally, visitors to both the grocery store and school often use cycling as their mode of transport. Cyclists access the building park in either a designated bicycle parking area adjacent to the building or on the sidewalk in the vicinity. In order to make deliveries, trucks are required to park in the 
traffic lane in front of the building. The delivery must then cross the adjacent bicycle lane situated between the truck and unloading area (see Fig. 1).

An elevated asphalt hump levels the street with the sidewalk; its purpose is to assist deliveries. This hump is occasionally used by cyclists to access the bicycle parking, initially raising safety concerns at the site. Additionally, the street configuration and traffic management in the area results in several trucks having to make a 3-point turn in order to turn around either before or after deliveries are made. Figure 2 illustrates the case study location.

The roadway in front of the building is a two-way street used by both motorised traffic and cyclists, with the northern adjacent block of the street transitioning to one-way for motorised traffic and two-way for cyclists. There are one-directional cycle lanes on both sides of the street in addition to sidewalks. Figure 3 shows the streetscape alongside the building. The speed limit is $30 \mathrm{~km} / \mathrm{h}$ and the area's function is residential. Based on 2013 traffic count data, the AADT is 2500 vehicles/ day, with $3 \%$ being trucks (vehicles longer than $5.6 \mathrm{~m}$ ) (www.vegvesen.no/vegkartreference). In the spring of 2016, a weekday cyclist count conducted within this case study found nearly 1400 cyclists using the roadway section between 6:00 and 18:00 with the highest volumes during the morning (7:00-9:00) and afternoon (15:00-17:00) peaks.

As previously mentioned, a safety evaluation of the site was initially proposed by the municipality after the report of a crash of a cyclist into the truck lift, which was located on the elevated hump in the street. It was reported by truck drivers that cyclists were using the elevated hump to access the sidewalk and cycle parking facility located next to the building. If there is a delivery truck parked there for unloading, the manoeuvre can result in a crash with the truck's lift that is placed on the elevated hump (see Fig. 4, green arrow represents cyclist's movement). Additionally, there is also the risk of conflict between cyclists riding in the cycle lane and drivers using a trolley to deliver pallets across the cycle lane (see Fig. 4 top, blue arrow). After an inspection of the premises, the Norwegian Labor Inspection Authority found the conditions for delivery workers hazardous and submitted a safety directive to the building's owner. In response to this ruling, rumble strips were added on cycle lanes in both directions to raise awareness of cyclists about the presence of delivery trucks. Additionally, a small sign and warning light were installed in order to warn cyclists of deliveries.

\section{Safety evaluation}

The original intention of the safety evaluation was to observe the street in the vicinity of the loading area before and after implementation of the rumble strips and to analyse their effect on the number and severity of conflicts between cyclists and delivery trucks. During a one-week observation period before the implementation of this measure, nearly no relevant encounters between trucks and cyclists were recorded in the loading area that could be compared using a before-after study. At the same time, safety issues related to truck-turning manoeuvres before or after deliveries were identified as a concern and further investigated. Thus, this research does not have a standard "before-after study" design. Instead, a safety evaluation of the entire section was conducted using video observations and intercept interviews. From these, safety risks at the site can be clearly identified.
Fig. 1 Freight delivery configuration as recommended by the receiver (position \#1 further in the text)

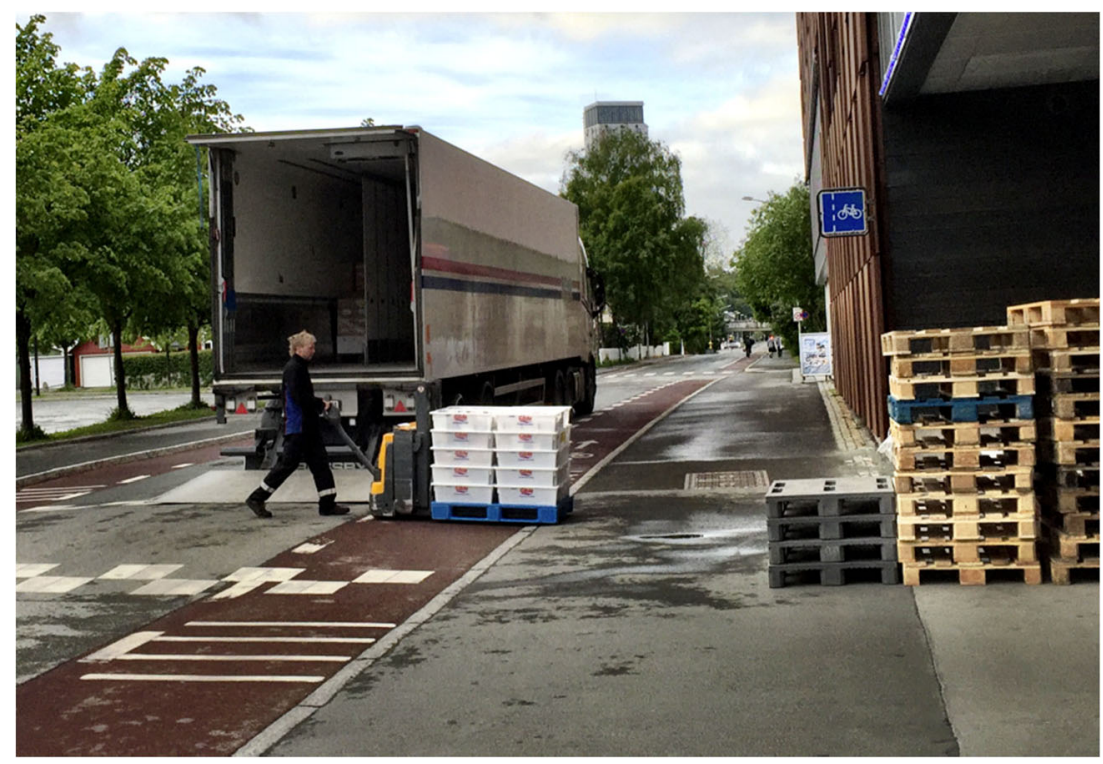


Fig. 2 Map of case study in Trondheim, Norway

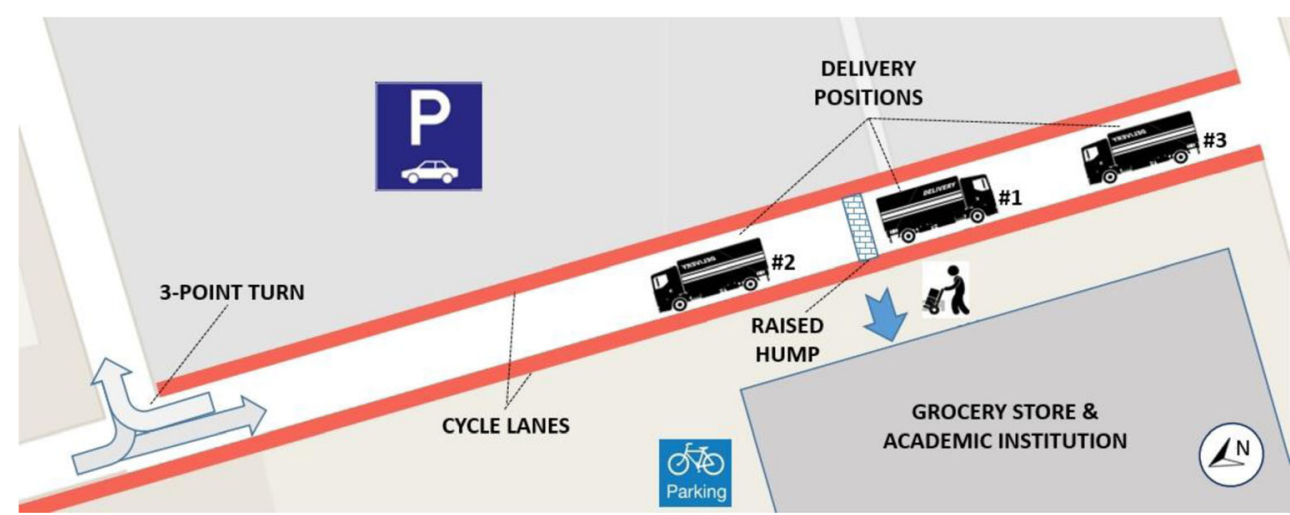

\subsection{Video recordings}

Using three camera locations (see Fig. 5), over $100 \mathrm{~h}$ of video was recorded to examine truck manoeuvres and delivery positions, safety levels between bicycles and delivery trucks, and behaviour of cyclists. As previously mentioned, while the original intent of the study was to examine behaviour at the loading zone itself, the videos revealed additional risk issues within the roadway section.

\subsubsection{Loading zone}

Camera \#1 recorded the loading zone for five working days (Mo-Fri; 7:00-17:00) before the implementation of the rumble strips, while camera \#3 recorded the behaviour of cyclists after their implementation for one working day (6:00 to $18: 00$ ), focusing specifically on cyclists riding across the rumble strips.

Fourteen cyclists were recorded by camera \#1 as having used the elevated hump to cross the road whenever there was a truck parked there (before rumble strip installation). No conflicts, indicated by cyclists' evasive action, were recorded during those manoeuvres. After the rumble strip installation,
Camera \#3 did not capture any observable/significant effect of the rumble strips on cyclists' behaviour. While a few cyclists chose to ride in the traffic lane, thereby avoiding any riding over rumble strips, this occurred very seldom (10 out of 1358 total cyclists) and does not necessarily relate to the presence of the rumble strips.

From the Camera \#1 recordings, observation of the coexistence between delivering trucks/trolleys and cyclists was also interesting. Eighteen trucks parked in position \#1 (see Fig. 2) when making a delivery to the building during the observation period, spending on average 19 min parked during each delivery. During this time, 165 cyclists rode in the cycle lane closest to the grocery store as they approached the parked truck. Three options were observed by cyclists when passing a truck parked in the loading zone:

1. Continue using the cycle lane

2. Riding around using the sidewalk

3. Riding around using the road

The choice of the passing manoeuvre is logically influenced by the position of the truck in relation to the cycle lane. When the truck was parked next to the cycle lane (as
Fig. 3 Streetscape view of case study site (source: adapted from Google maps, 2016)

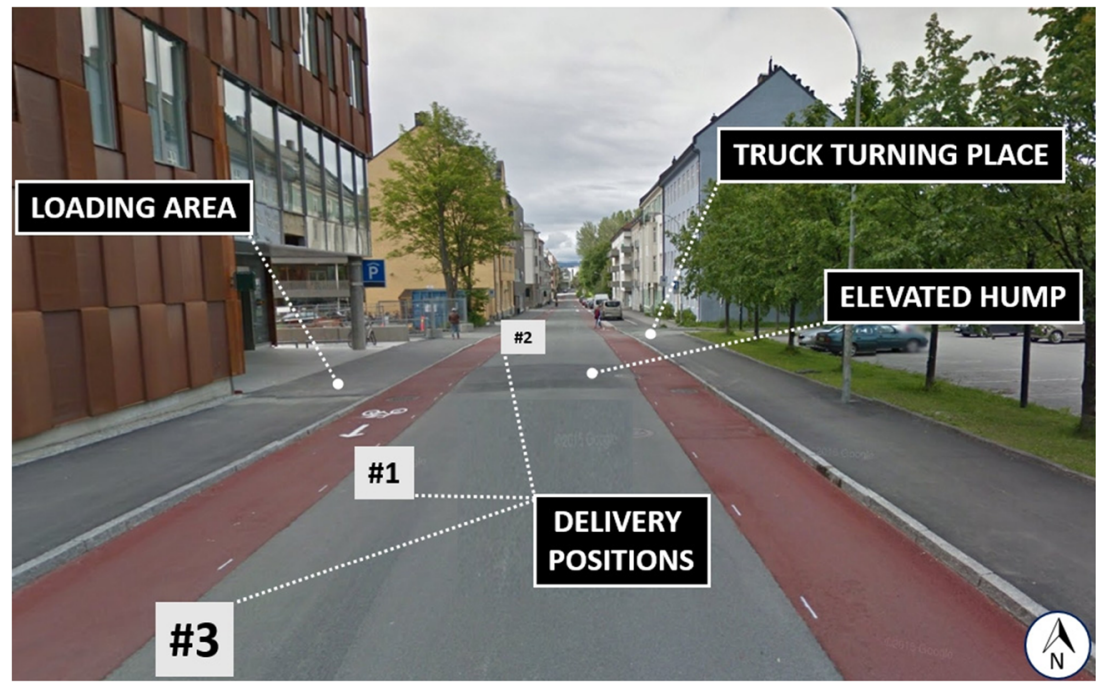




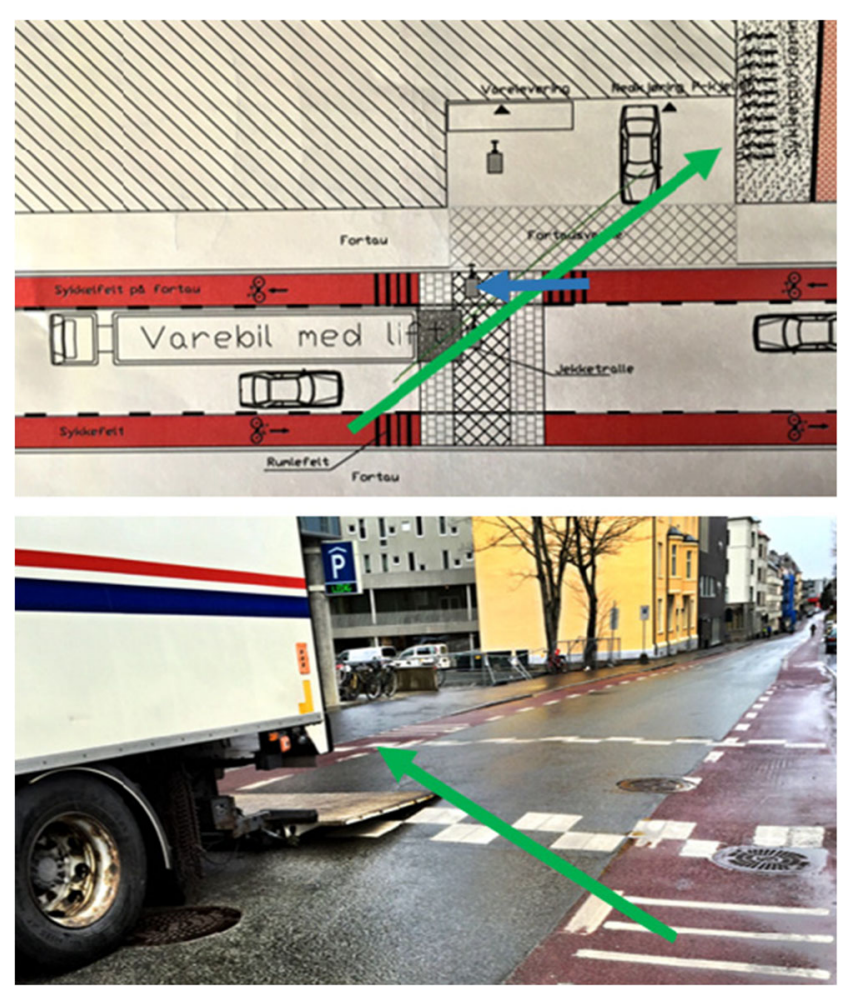

Fig. 4 Scheme of the proposed safety measure and risky manoeuvre (green arrow). Implemented measure on bottom picture

it should be), nearly all cyclists continued to use the cycle lane. When the truck occupied the cycle lane (just 1 occurrence), half the cyclists used the sidewalk and half used the traffic lane in order to go around the truck. There were two recorded conflicts (an avoidance manoeuvre by the cyclist) between cyclist riding in the cycle lane (in the "correct" direction) and delivery trolleys.

While travel by cyclists in the contra-flow direction was not common (approximately 6\%), it was identified as a concern. It is "normal" behaviour for cyclists, who start their trip from the building and continue north towards the city centre. Nevertheless, this behaviour is not easily accepted by truck drivers, as deliveries using trolleys become more obscured when cyclists are riding contra-flow. However, no conflict was observed in these situations.

\subsubsection{Truck turning Manoeuvres}

The recordings from cameras \#1 and \#2 also highlighted several types of truck manoeuvres performed in the studied section. All trucks enter the section through the same intersection and then park in one of three different positions (see Fig. 2). Seventy percent of trucks also leave the roadway section through the same intersection; thus they must complete a 3point turn manoeuvre either before or after making a delivery. This manoeuvre is conducted in the entrance to the parking lot on the other side of the street (see Fig. 2 for location), and the truck must cross over both cycle lanes to make the turn.

During observations over the five working days, 35 trucks made deliveries to the building, with 24 trucks making the previously described 3-point turn manoeuvre. Twenty-eight cyclists rode through the road section during those manoeuvres, with nearly half of them needing to react in some way based on the trucks' movements. These reactions included riding in the opposite traffic lane, going around the reversing truck, and waiting in the cycle lane while the truck was reversing. Two conflicts, both based on an evasive action, were recorded. One concerned a truck leaving the car park whose driver noticed a cyclist riding in the cycle lane from the left too late and had to brake suddenly, and another where a truck driver came to an abrupt stop to accommodate a cyclist who was crossing the road diagonally in front of the truck.
Fig. 5 Locations of camera and areas of interest

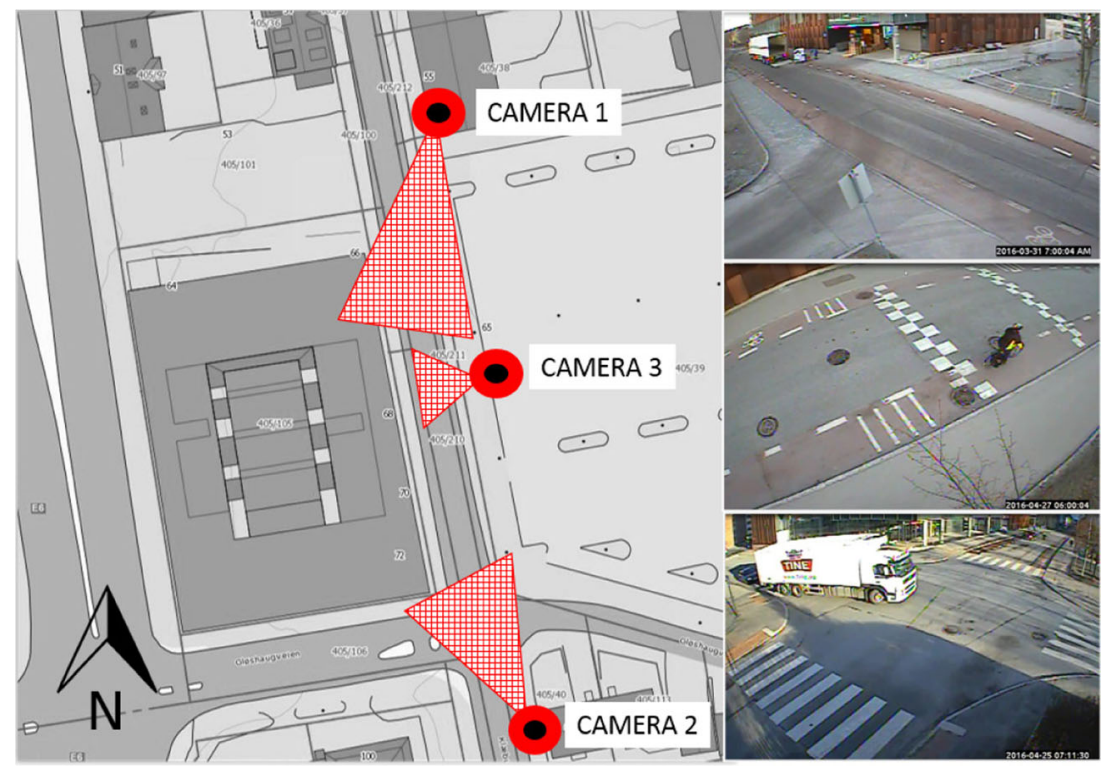




\subsection{Intercept interviews}

As the main purpose of the installation of the rumble strips was to raise cyclists' awareness of the presence of delivery trucks, intercept interviews were conducted after the measure's implementation to determine if cyclists noticed the measure and if they understood its purpose. Several truck drivers were also interviewed in order to provide further insight into the situation. Cyclists who rode across the rumble strips and stopped near the grocery store and truck drivers who delivered goods and parked in the section were interviewed. The interviewer asked the following questions of each group:

\begin{tabular}{ll} 
CYCLISTS: & TRUCK DRIVERS: \\
$\begin{array}{ll}\text { 1. How often do you cycle on } & \text { 1. What parking position do you } \\
\text { this section of roadway? } & \text { prefer when delivering to the } \\
\text { 2. Were you aware of the addition } & \text { store and why? } \\
\text { of the recent rumble strips in } & \text { 2. Is there any recommendation } \\
\text { the cycle lanes? } & \text { from the store regarding what } \\
\text { 3. What do you think is the purpose } & \text { position to choose? } \\
\text { of the rumble strips? } & \text { 3. Do you think the recently added } \\
\text { 4. Have the rumble strips changed } & \text { rumble strips in the cycle lanes } \\
\text { your cycling behaviour? } & \text { will reduce the conflicts with } \\
\text { 5. Have you experienced any } & \text { bicycles while you are } \\
\text { safety problems with trucks } & \text { unloading? } \\
\text { at this site? } & \text { 4. Have you experienced any } \\
& \text { safety problems with bicyclists } \\
& \text { at this site? }\end{array}$ \\
\hline
\end{tabular}

Thirty-nine cyclists were interviewed. Most respondents were adults who cycle frequently in the area (3-5 times in a week). A truck was presented in $33 \%$ of the intercepts. Only $28 \%$ of the respondents noticed the presence of rumble strips, and $13 \%$ said they changed their behaviour due to the implementation. The majority (80\%) thought that the rumble strips' main purpose/function was to slow down cyclists. No respondents connected the rumble strips directly with the delivery trucks, but $23 \%$ thought that the rumble strips were implemented as a general warning to increase cyclists' alertness. Interestingly, no cyclists commented on the risk associated with the turning manoeuvres of trucks that was noted in the video recordings. There were significantly fewer comments about delivery trucks when there were no trucks present during the interview.

Five truck drivers were interviewed. Four parked in position \#1 (see Fig. 2). All drivers said that this position is recommended by the grocery store because it is closest to the loading dock and does not block the entrance to the garage. The majority of drivers were sceptical about the effect of rumble strips on reducing conflicts with cyclists. Four drivers parked their truck adjacent to the cycle lane. One driver parked in the cycle lane (which is illegal), stating this was done on purpose to avoid conflicts with cyclists during their delivery. Drivers highlighted having difficulties with cyclists during turning manoeuvres and loading operations. Two drivers mentioned the issue of cyclists riding in the cycle lane in a contra flow-direction, which added to the complexity of the situation.

\subsection{Identified safety risks}

The safety evaluation of the case study site identified several potential risks with unknown magnitudes associated with truck deliveries and cyclist movements. These include:

- Potential crashes of cyclists crossing the road (using the elevated hump) with the truck's lift (the catalyst for the study)

- Conflicts between cyclists and delivery trolleys, especially with cyclists who ride contra-flow

- Conflicts between cyclists and other traffic, if cyclists are unable or afraid to use the cycle lane and go around the truck in the traffic lane

- Trucks or cars driving in the opposite direction and interfering with the cycle lane because another truck is parked there to make a delivery and does not provide enough space for them to pass in the driving lanes

- Conflicts between cyclists and trucks that are performing 3-point turning manoeuvres

- Conflicts between cyclists and cars entering/leaving the underground parking when a truck parked on the road obscures visibility

The addition of the rumble strips and warnings lights attempts to address the safety risk associated with cyclists using the elevated hump, although its efficacy is in doubt, as noted in the interview results and observations. At the same time, the observations did not identify this manoeuvre as being the most critical safety issue when considering numerous additional safety risks identified above. Conflicts related to visibility issues, particularly to blind spots during truck manoeuvres, which have been identified as one of the main risk factors in truck and bicycle accidents $[6,7,19-21]$ are thus of great safety concern.

\section{Mapping of planning process within the case study}

While only one truck-bicycle accident has been recorded at the location thus far, the observations of the truck-bicycle encounters revealed several areas of safety concern. From the safety analysis and subsequent identification of safety risks, it is obvious that encounters between street users (namely cyclists and delivery trucks in this case) were not considered in either the site or street infrastructure design. Given the expected volumes of trucks and bicycles on this street section, it seems obvious in retrospect that the coexistence between these user groups should have been examined in more detail during planning phases. To better understand why this issue 
was not considered, the planning process associated with the building design and construction was investigated.

As part of a larger project, Sustainable Urban Logistics Plans in Norway (NORSULP) [22], empirical data was collected in order to better understand and map the planning process. For this specific study, we base our findings on: (1) a study of how laws and thematic policy plans for the shared urban space interact with current protocols for freight delivery, and (2) semi-structured interviews with stakeholders in the case, including representatives from the local authorities, e.g., heads and managers of urban planning and case officers, as well as non-governmental representatives, e.g. project owners, architects, and the freight industry. These interviews focused on the different perspectives, priorities, involvement, and interaction the different stakeholders had throughout the specific case leading up to the present situation. Publiclyavailable case documents and correspondence were used to identify the interviewees, who were selected based upon their roles and experience within urban logistics and the case study project. Six interviews were conducted either in person or over the phone. The questions were open-ended, allowing the interviewees to address and discuss topics they personally thought were relevant for the case. The interviews addressed two overarching themes. First, we enquired about their roles and responsibility in relation to different aspects of urban freight logistics. Second, we asked open-ended questions about their involvement in the specific case and how they perceived the collaboration and communication across departments, plans, and between stakeholders to be. We did not interview any politicians, as this case study aimed to explore the implementation of policies already in place.

\subsection{Timeline of the planning process}

Based on the case documents and interviews, we mapped the timeline of events associated with the design and construction of the building, changes to the roadway, and freight delivery decisions, in turn relating these events to the planning context and current procedures (Fig. 6).

The bicycle plan developed in 1998 was a thematic plan whose intention was to identify the main bicycle routes that were planned across the city. The street in this case study was identified as being one of the streets where the planned route would go. However, the thematic plan did not specify when the construction of the bicycle infrastructure was to begin or how the infrastructure was going to be designed and laid out along each street. So while the thematic plan was not legal in any sense, it merely served as a guideline.

After the initiative for a new grocery store on the site was set in motion by the developer (2010), building construction plans were designed in collaboration with an architect. Next, a designated case officer arranged an obligatory start-up meeting at the city planning office, which presented an arena for the developer to display their plans and discuss with city planning office representatives, among other things, contextual issues about the construction and planned operation of the building. It is not known if issues related to truck deliveries were discussed in this meeting, but at the time there were no city planning department employees who were specifically tasked to consider freight and/or goods movement in the building's planning process.

The central directive for all construction in Norway is the Plan and Building Act. Although the law contains paragraphs relevant to freight delivery, it does not consider delivery situations directly. Directives for freight delivery solutions must therefore be interpreted from general statements found in the law. To support local planners and developers, organisations such as the National Public Road Administration (NPRA) have developed detailed 'best-practice guidelines'. However, these guidelines do not focus on shared urban space for the street users or general delivery isues; instead, they are mainly concerned with technical aspects of the construction plans to ensure a safe environment for workers. Establishment of delivery ramps are evaluated with the current street plans. Because the Plan and Building Act does not require a detailed plan for the building's operation, the project owner, in collaboration with an architect, may initiate the building process of commercial real estate without explicitly knowing what store(s) will reside in the building. This means that from the time the plans are initiated until the construction project is finished, the expected type and frequency of goods delivery may have changed. In the case presented here, the planned use of the building (grocery store) was already determined. However, since the planned use of commercial real estate may change from initiation to operation, start-up meetings typically focus only on the plan's construction.

After the start-up meeting, the building plans were announced officially, and stakeholders such as neighbours and the freight delivery union were notified in writing. Stakeholders were given the opportunity to raise concerns about the building's design and planned operation. In this case, no external stakeholders made any remarks about the plans. When concerns are raised, there may be revisions to the plan and subsequent public hearings. Once the plans were accepted in the review process, the case officer presented the construction plans for the city council. They approved the plan, and construction began (2013). There were no evident discussions regarding freight deliveries at the building location within the review process or approval by the city council.

During the building planning process, the city council announced a thematic 'Green City Plan'. These thematic plans are most often non-juridical and lack the level of detail useful for individual building plans. One of the main priorities of the Green City Plan was to offer continuous bicycle paths throughout the city. In conjunction with the 'Green City Plan', the city council decided to move forward with the 
Fig. 6 Timeline of events in the planning process
-A non-juridical bicycle plan for Trondheim is announced. Main routes are identified at street level.

- Building construction plans initiated by developers

- Start-up meeting with stakeholders, including city planners

-Plans are announced to relevant stakeholders with hearing rights

- Detailed building design plans developed by architects

- Revision by city planners

- Public hearing (no revisions were required)

- The Green City Plan is announced by the city council. One of the priorities in the plan is seamless bicycle routes throughout the city.

- Building construction plans are completed and submitted to case officer for review

- Case officer submits plan to city council, which approves plan

2012

- Building construction begins

2013

- Building construction finishes, building becomes operational

- Trondheim approves a new bicycle strategy, and bicycle lanes in both directions are created along the street in front of the building with incentives from the Green City Plan

- City planners announce a change in the adjacent street section from a two-way to a one-way

- After a complaint from the freight delivery union, the Norwegian Labour Inspection Authority finds conditions for freight delivery personnel hazardous due to bike lanes crossing path of delivery (Fig. 1 and 2). The owner of the building is responsible for improving the condition. - Rumble strips are placed on the road, but there are still doubts as to the efficiency and safety of the delivery situation bicycle plan from 1998, which included the construction of bicycle infrastructure along the street section in the case study. While the bicycle plan contained the planned route, a final decision on the specific street was not implemented until 2014. In addition, the plans did not specify the design of these paths. It is uncertain to what degree, if any, the bicycle plan was updated to reflect changes in the city's development in the 10 -plus years since its establishment. Although, with respect to this case study, it is unlikely that there would be any change to the placement of a bicycle route along the road section given that it is a logical link within the bike network with limited route alternatives within the corridor. After the building construction was complete and building became operational, bicycle lanes were installed on either side of the street.

Additionally, after the construction was completed, city planners in collaboration with regional road authorities set in motion a new street plan that altered the road section just north of the building site from a two-way street to a one-way, in effect limiting the mobility of larger trucks. The new street plans were under development at the time the construction project had started. Based on our conversations with the interviewees, it appears that neither the bicycle lane plans or changes in the street configuration were addressed by any of the public stakeholders until after the building was finished and operational and changes to the streetscape were completed.

After complaints were filed by the freight delivery union (LUKS), the Norwegian Labour Inspection Authority determined that conditions for freight delivery personnel were hazardous due to the bike lane crossing the path of delivery. The building owner and the city planning office decided to install the previously discussed rumble strips to alert cyclists to the presence of delivery trucks (and perhaps slow down bicyclists crossing the delivery zone, although this was not the direct intention). Several months after the rumble strips were installed, a small sign and flashing light were also installed to warn cyclists of impending deliveries.

\section{Discussion}

The technical safety evaluation clearly identifies numerous risk factors associated with truck deliveries and bicycle mobility at the site investigated within this research, while the planning process evaluation highlights the fact that a strategy for freight delivery is lacking for both this specific case study and overall within the city. Together, these evaluations illustrate how the lack of concern for urban freight deliveries within urban planning can result in risky situations for road users. In mapping the planning process, no discussions of freight delivery were identified. The standard planning process is based on laws and regulations which do not include any requirements to do so. Today, urban freight is treated on a case-by-case basis, and if any strategies or objectives concerning urban freight do exist, they are fragmented, which implies that there is not necessarily coordination within or between projects. Thus, even when freight delivery is discussed within a project, there is likely to be a lack of communication and coordination between the different city planning departments involved. 
This lack of coordination is evident in the case study, as the city planning office held three separate roles here: (1) responsibility for reviewing and approving building construction plans, (2) responsibility for planning and implementing new bicycle infrastructure, and (3) responsibility for planning and implementing a change in the street function, with minimal to no interaction among these three roles. Additionally, urban freight transport was not considered within any of these roles, despite the inherent need for deliveries given the function of the building. While such coordination can be challenging due to the complexity of city administration, the current set-up provides no opportunity for urban freight transport considerations. The planning timeline indicates lack of coordination between the involved departments, lack of knowledge about how the different aspects of both building and street design impact urban freight, and a lack of focus and strategy related to urban freight transport.

As observed in this case study, the lack of coordinated urban planning resulted in safety concerns in which the delivery situation could potentially result in severe conflicts between trucks and cyclists. In examining the technical solution to address the safety concerns, there are doubts about the measure's effectiveness, further highlighting the lack of understanding of both bicyclists' and truck drivers' needs. The intent of the rumble strips was to raise awareness of deliveries, which is not necessarily evident according to the cyclists and truckers interviewed, especially given their location directly adjacent to the elevated hump. While the rumble strips may slightly reduce cyclists' speeds, which may in turn reduce the potential for or severity of encounters, they also serve as a potential hazard in the lane and may cause additional safety issues (for example, friction issues when the road and markings become wet). Additionally, the risk perceived as high by the municipality differs from those risks identified within safety observations. This discrepancy not only further highlights the lack of understanding regarding mobility at the site, but also clearly demonstrates the importance of the observation method, as it can reveal safety problems that would otherwise remain hidden if only accident data is considered.

Regardless, it is challenging to identify an effective technical solution to improve the delivery situation given the constraints of the completed building and streetscape given the fact that the finished construction project and changes to the delivery configuration are largely restricted to small, cosmetic implementations. This further highlights the importance of discussing freight and delivery issues early in the planning process, when there is still the possibility to make more substantial changes to the designs. This proactive approach is also recommended with regard to road safety in a general sense, where it is suggested that trying to address safety concerns before infrastructure is designed - as opposed to the reactive improvement of deficiencies - will result in an overall better road system [23]. Additionally, while the costs associated with the road construction were small, especially when compared to the costs associated with the building construction, these road constructions are funded through tax-payer contributions. Thus, there is a need for road modifications to address deficiencies soon after initial construction plans are made, as this action may indicate a misuse of these funds.

While there is a mobility plan within Trondheim [24] which attempts to unite planning and transport efforts with the goal of environmental sustainability, it only involves personal mobility by public transport, walking and biking. Any mention of urban freight policy for goods and services is lacking here, and there are no personnel responsible for this task at the city administration level. A freight plan (or a SULP) would be a useful planning tool for the city administration to increase awareness of freight delivery issues as well as identifying and addressing problems similar to that found in this case study. The establishment of this framework would allow for both meaningful interactions between stakeholders and the development of best practices related to urban freight deliveries. The long-term dialogue between stakeholders that can be developed through such a framework can be compared to urban freight partnerships, which have been shown to result in stronger relationships between stakeholders, improved communication and knowledge sharing, and improved decision-making [25]. For the case examined within this study, earlier discussions of deliveries between both private and public stakeholders may have resulted in requiring the building design to include an off-street delivery dock, the use of alternative bicycle infrastructure designs, or consideration of alternative operations such as overnight deliveries. As further development is expected along this street, it is recommended that these discussions take place early in the planning process in order to avoid further exacerbation of mobility and safety issues.

\section{Conclusions}

In the absence of a dedicated freight policy and/or personnel at the governing authority, it is entirely up to the planner, architect, and developer to ensure good solutions for freight delivery. The lack of planning regulations and specifications regarding freight delivery solutions results in situations where urban freight concerns are commonly discussed far too late in the planning process - or sometimes not at all - often to the detriment of all road users. Numerous safety risks were identified while observing delivery operations in this particular casy study which were the result of limited discussions of freight during the planning process and lack of coordination among various city planning departments. Post-construction mitigation efforts are limited. Early detection of delivery issues through closer cooperation between plans and planners may enable more meaningful corrective action early on in the process. This coordination can also ensure that various plans (construction, street, thematic) are considered holistically and 
with consideration to other existing plans and development in the area. However, without any dedicated city plans for how freight delivery fits into the shared urban space, current deficiencies in the planning process will likely lead to continued problematic and potentially dangerous environments.

Open Access This article is distributed under the terms of the Creative Commons Attribution 4.0 International License (http:// creativecommons.org/licenses/by/4.0/), which permits unrestricted use, distribution, and reproduction in any medium, provided you give appropriate credit to the original author(s) and the source, provide a link to the Creative Commons license, and indicate if changes were made.

\section{References}

1. Manson J, Cooper S, West A, Foster E, Cole E, Tai NRM (2012) Major trauma and urban cyclists: physiological status and injury profile. Emerg Med J. doi:10.1136/emermed-2011-200966

2. Kaplan S, Vavatsoulas K, Prato CG (2014) Aggravating and mitigating factors associated with cyclist injury severity in Denmark. J Saf Res 50:75-82. doi:10.1016/j.jsr.2014.03.012

3. Kröyer HRG (2015) The relation between speed environment, age and injury outcome for bicyclists struck by a motorized vehicle - a comparison with pedestrians. Accid Anal Prev 76:57-63. doi:10. 1016/j.aap.2014.12.023

4. International Transport Forum (2013) Cycling, health and safety. OECD Publishing, Paris

5. Pokorny P, Drescher J, Pitera K, Jonsson T (2017) Accidents between freight vehicles and bicycles, with a focus on urban areas. Trans Res Proc. doi:10.1016/j.trpro.2017.05.474

6. Johannsen H, Jänsch M, Otte D, Urban M (2015) Accidents involving turning trucks and bicyclists - options for analysing countermeasure. Paper presented at international cycling safety conference. Hannover, Germany

7. Seiniger P, Jost G, Benjamin S (2015) Development of a test procedure for driver assist systems addressing accidents between right turning trucks and straight driving cyclists. Paper presented at the 24th international technical conference on the enhanced safety of vehicles (ESV), Gothenburg, Sweden

8. Pivo G, Carlson D, Kitchen M, Billen D (2002) Learning from truckers. J Archit Plann Res 19(1):12-29

9. Bassok A, Johnson C, Kitchen M, Maskin R, Overby K, Carlson D, Goodchild A, McCormack E, and Wygonik E (2013) NCFRP report 24: smart growth and urban goods movement. Transportation Research Board of the National Academies, Washington, D.C., 21
10. Conway A, Thullier O, Dornhelm E, and Lownes N (2013) Commercial vehicle-bicycle conflicts: a growing urban challenge. Presented at 92nd annual meeting of the Transportation Research Board, Washington, D.C., 22

11. Gelino K, Krass C, Olds J, Sandercock M, and Bassock A (2012) Why Can't we be friends? Reducing conflicts between bicycles and trucks. Studio report, Department of Urban Design and Planning, University of Washington, Seattle. http://faculty.washington.edu/ abassok/studio/reducing_conflicts_between_bicycles_and_trucks. pdf. Accessed 27 July 2016

12. Allen J, Thorne G, and Browne M (2007) BESTUFS: Good Practice Guide on Urban Freight Transport http://www.bestufs. net/download/BESTUFS II/good practice/English BESTUFS Guide.pdf. Accessed 1 Dec 2016

13. Norwegian Public Road Administration (2005) Handbook V126, The City and Goods Transport (in Norwegian). Vegdirektoratet, Oslo

14. Trondheim Kommune (2006) Street Use Plan for the Center City 2007. https://www.trondheim.kommune.no/attachment.ap?id= 48964. Accessed 12 Dec 2016

15. European Commission (2013) A call to action on urban logistics. Commission Staff Working Document SWD 524, final. Brussels

16. Ambrosino G et al (2014) The role of logistics Services in Smart Cities: the experience of ENCLOSE project. Proceedings of REAL CORP, Vienna, Austria. ISBN: 978-3-9503110-7-5

17. Spurkeland E and Andersen J (2014) Urban logistics plans are needed Urban logistics has been neglected (in Norwegian). Samferdsel 7/2014. https://samferdsel.toi.no/nr-07/varetransport-ibyene-article32662-1463.html. Accessed 10 Oct 2016

18. Hjorthol R, Engebretsen Ø, Uteng TP (2014) 2013/14 National travel survey - key results. Institute of Transport Economics. https://www.toi.no

19. Niewoehner W, Berg A (2005) Endangerment of pedestrians and bicyclists at intersections. Statistics:1-15 http://www-nrd.nhtsa.dot.gov

20. Sagberg F, Sørensen M (2012) Road safety in Norwegian urban areas - accident analysis and assessment of countermeasures. Institute of Transport Economics. https://www.toi.no

21. Volvo Truck (2013) European Accident Research and Safety Report 2013. http://www.volvotrucks.com

22. NORSULP (2016) Sustainable Urban Logistics Plans in Norway www.norsulp.no (in Norwegian)

23. de Leur P, Sayed T (2003) A framework to proactively consider road safety within the road planning process. Can J Civ Eng 30: 711-719. doi:10.1139/L03-034

24. Miljøpakken (2016). Miljøpakken. Trondheim Kommune. http:// miljopakken.no/ (in Norwegian)

25. Lindholm M, Browne M (2013) Local Authority Cooperation with Urban Freight Stakeholders: A Comparison of Partnership Approaches. Eur J Transp Infrastruct Res 13(1):20-38 